\title{
Periodontal Vaccine: A New Paradigm for Prevention of Periodontal Diseases
}

\author{
Nikhil Sharma', Nitin Khuller
}

\begin{abstract}
Vaccination is a process that induces specific immune resistance to a bacterial or viral infectious disease. Vaccines have prevented several infectious diseases for many years, and are still being investigated. In late eighteenth century, Edward Jenner developed and established the principle of vaccination using the cross protection conferred by cowpox virus, which is non pathogenic in humans.

Regarding a vaccine against the periodontal disease, the complexity of the periodontopathic bacteria might be a problem in determination of Antigens. Among some 300 species of bacteria involved in subgingival plaque, 5-7 species have been implicated in the etiology of periodontitis but one or two species; P.gingivalis or B. forsythus might play an important role as primary pathogens.

Vaccination accomplished can be active immunization, passive immunization or DNA vaccination, made from the antigenic epitopes in periodontopathic bacteria. In light of the increasing evidence that periodontitis significantly increases risk for potentially fatal diseases such as coronary heart disease, stroke and complications from diabetes mellitus a successful vaccine for periodontitis could have health benefits far exceeding the prevention of periodontitis.
\end{abstract}

KEYWORDS: Vaccination, Periodontitis, Periodontopathic, Immune response, DNA vaccine.

${ }^{1}$ MDS

Dept. of Periodontics

I.T.S Dental College and Hospital,

Murad Nagar, Delhi- Meerut Road

Ghaziabad-201206 (U.P)

Email: nikhilsharma31@gmail.com

${ }^{2} \mathrm{MDS}$

Reader

Dept. of Periodontics

I.T.S Dental College and Hospital,

Murad Nagar, Delhi-Meerut Road

Ghaziabad-201206 (U.P)

\author{
Contact Author \\ Dr. Nikhil Sharma \\ E-mail : nikhilsharma31@gmail.com \\ J Oral Health Comm Dent 2010;4(Spl)23-28
}

\section{INTRODUCTION}

$\mathrm{V}$ accination is the best-known and the most important application of immunological principles to human health (1). The first vaccine was named after vaccinia, the cowpox virus. Jenner pioneered its use 200 years ago. It was the first deliberate scientific attempt to prevent an infectious disease (small pox). But it was done in complete ignorance of viruses (or indeed any kind of microbe) and immunology.

It was not until the work of Pasteur 100 years later that the general principle Governing vaccination emerged that altered preparations of microbes could be used to generate enhanced immunity against the fully virulent organism. Thus, Pasteur's dried Rabies-infected rabbit spinal cords and heated anthrax bacilli were the true forerunners of today's vaccines, while Jenner's animal-derived (i.e. heterologous) vaccinia virus has had no real successors.
Even Pasteur did not have a proper understanding of immunological memory or the functions of the lymphocyte, which had to wait another half century. Finally with Burnet's clonal selections theory (1957) and the discovery of $\mathrm{T}$ and $\mathrm{B}$ lymphocytes (1965), the key mechanism became clear (1).

Vaccination against bacterial and viral infectious diseases has progressed immeasurably throughout twentieth century. For many diseases, this has been achieved with brilliant success, but for others there is no vaccine whatsoever.

\section{PRINCIPLES OF VACCINATION}

The principle of vaccination is based on two key elements of adaptive immunity namely specificity and memory (1). The antigen(s) of a vaccine induces clonal expansion in specific $\mathrm{T}$ and/or $B$ cells leaving behind a population of memory cells. These enable the next encounter with the same antigen(s) to induce a secondary 
response which is more rapid and effective than the normal primary response. (Fig. 1)

The aim in vaccine development is to alter a pathogen or its toxins in such a way that they become innocuous without losing antigenicity. This is possible because antibodies and $T$ cells recognize particular parts of antigens, the epitopes, and not the whole organism or toxins. (Fig. 2)

\section{Key features of a successful vaccine}

- It should be safe to administer

- It should induce the right sort of immunity

- Vaccine should be effective against the particular infectious agent and prevent the disease

- It should be stable and have a long shelf life.

- Vaccines should be affordable by the population at which they are aimed.

\section{Immunization against periodontitis}

A substantial number of bacteria (exceeding 300 species) appear to be involved in subgingival plaque. Among these five to seven species have been implicated in the etiology of periodontitis, but one or two species, $\mathrm{P}$. gingivalis or $\mathrm{B}$. forsythus, might play an important role as primary pathogen $(2,3)$. Furthermore, regarding antigenic epitopes in periodontopathic bacteria some researches have demonstrated that epitopes were shared among gram negative bacteria, possibly because of the polyclonal B-cell activating properties of lipopolysaccharide.

Several investigations regarding the humoral immune response in periodontitis patients have been performed. Chen et at. 1991, reported that 24 out of 36 rapidly progressive periodontitis patients were seronegative to antigens of $\mathrm{P}$. gingivalis and both serum antibody titers and avidity against P.gingivalis antigens from 24 seronegative patients increased significantly following only scaling and root planning. Similar observations were made by Sjostrom K et al. 1994, on groups of subjects with rapidly progressive periodontitis tested for antibodies against A actinomycetemcomitans. Ten of 12 patients became seropositive from seronegative following scaling and root planing, and the post-treatment sera enhanced stronger capacity of phagocytosis and killing than the pretreatment ones did. Periodontal therapy, scaling and root planning, could elicit the humoral immune response in seronegative patients, resulting in seroconversion and production of effective antibodies. This might be due to bacteremia provoked by treatment (4).

These observations suggest that the development of vaccine against periodontitis might be possible and that utilization of it could be an effective method for control and prevention of periodontal disease.

\section{EXPERIMENTAL MODELS}

Humans may not be used as experimental subjects in studies periodontopathic bacteria. Accordingly, the prevention of colonization, adhesion and bacterial invasion has been studied in various animal models. Non human primates \& humans are similar in both periodontal structure \& microflora composition. However, ligatures must be tied around the teeth to elicit periodontitis in nonhuman primates, because it is difficult to colonize the oral cavity with P.gingivalis and establish periodontal lesions.

Studies in non-human primate models using ligature-induced experimental periodontitis suggest that antibody responses by active immunization against Porphyromonas gingivalis can safely be induced, enhanced, and obtained over time (5).

McArthur et al. suggested that the squirrel monkey could be used as a model for studying the parameters of blackpigmented anaerobic rods colonization in gingival crevices (6). However, the mechanisms of bacterial retention around ligated teeth are totally different from those of adhesion around the teeth or gingival tissue.

Persson et al. investigated the constituents of subgingival microflora and immune reactions (antibody titers and avidities against $\mathrm{P}$. gingivalis) in experimental Macaca fascicularis periodontitis and concluded that $\mathrm{M}$ fascicularis was a useful model for testing and developing vaccines for periodontitis (7).

There are some advantages in using rats for adhesion experiment. Since rats resemble humans in periodontal anatomy and bacterial composition, bone loss can be evaluated $(8,9)$. Furthermore, P.gingivalis quickly colonizes the rat oral cavity and induces bone loss. The invasion ability of bacteria has been investigated using the subcutaneous abscess model in mice and the subcutaneous chamber model in mice and rabbits. Kesavalu et al. studied active immunization using whole cells or selected cell envelope components and suggested that the murine model would be useful for investigating the tissue-destructive components of P.gingivalis (10).

\section{ACTIVE IMMUNIZATION}

Active immunization has been studied using whole bacterial cells, outer components or synthetic peptides as antigens. The results showed that the progression of periodontal diseases could be prevented by immunization.

Natural active immunization by therapeutic interventions results in antibody titre enhancement and potentially improves treatment outcomes. Data suggest that immunization reduces the rate and severity of bone loss. It 
is also, temporarily, possible to alter the composition of the subgingival microflora (5).

\section{Antigen: Whole cells}

Klausen et al. reported that the levels of serum antibodies to both whole cells and partially purified fimbriae from P.gingivalis were elevated in rats immunized with P.gingivalis cells and that the activities of collagenase and cysteine proteinases in gingival tissues as well as periodontal tissue loss were decreased (11). Genco et al. demonstrated that protection against invasion but not colonization by P.gingivalis was induced in the mouse chamber model by immunization with either killed heterologous invasive or heterologous non-invasive P.gingivalis strains (12). Kesavalu et al. indicated that in the BALB/cmouse model, the immune response to whole cells or selected cell envelope components did not completely abrogate lesions but eliminated mortality (10). The numbers of P.gingivalis on ligatures in hamsters were reduced, and spreading infection was prevented by the subcutaneous administration of whole formalin-killed cells but not by a perioral injection (13). In squirrel monkeys (Saimiri scuireus), immunization with whole cells of monkey isolate (P.gingivalis strain I-372)increased the level of antiP.gingivalis IgG antibody in serum and significantly reduced colonization by the same strain in gingival crevices (6). Furthermore, Persson et al. reported that immunizing M fascicularis with killed P.gingivalis in Syntex Adjuvant Formulation-M inhibited the progression of periodontal tissue destruction (14).

Mice vaccinated subcutaneously with an inactivated, wholecell vaccine preparation of Porphyromonas denticanis, Porphyromonas gulae, and Porphyromonas salivosa displayed significantly reduced alveolar bone loss in response to heterologous and cross-species challenges as compared to sham vaccinated animals(15).

Page RC used ligature-induced periodontitis in the nonhuman primate Macaca fascicularis as a model, 10 animals were immunised using intact killed $\mathrm{P}$. gingivalis and SAF$\mathrm{M}$ adjuvant and 10 controls using adjuvant only (16). The vaccine, containing 250 microg protein/ml, was injected subcutaneously in the neck and into the deltoid muscle (0.5 ml each site) at baseline and weeks 3, 6, and 16, and the mandibular posterior teeth ligated at week 16. At weeks 30 and 36 changes in alveolar bone, measured using digital subtraction radiography, were used as the outcome measure. Even though periodontitis in humans and in this animal model is a polymicrobial disease, immunisation with a vaccine containing a single bacterial species, $\mathrm{P}$. gingivalis, induced protection. Of all the P. gingivalis components that have been studied, the cysteine proteases have the greatest potential as vaccine antigens. In a pilot study using the same protocol, it was shown that porphypain-2 purified from P. gingivalis is effective in inducing protection. In neither the whole cell vaccine nor the purified cysteine protease vaccine studies were signs of toxicity observed.

However, active immunization with whole cells might induce exaggerated inflammatory responses in the host. Bone density was significantly decreased in ligated teeth from nonhuman primates immunized with whole-cell antigens of P.gingivalis and P.intermedia (17). The investigators concluded that broad-based immune response to several bacteria antigens could increase hypersensitivity reactions to the bacteria.

\section{Antigen: Outer components}

Fimbriae from P.gingivalis play an important role in adhesion to oral tissues (18) and are also highly immunogenic $(11,18)$. Therefore, the effect of immunization with various fimbrial and cell surface antigen preparations upon P.gingivalis-associated periodontal diseases was investigated (19). Evans et al. reported that immunization with highly purified P.gingivalis fimbrial preparations $(43-\mathrm{kDa}$ fimbrial component), as well as with whole cells and soluble antigens of P.gingivalis, protected against periodontal destruction induced by P.gingivalis is in gnotobiotic rats (11). Gingival tissue enzymes, including collagenase and the cysteine proteases, cathepsin Band L, were markedly affected by immunization with these, antigens. They suggested that fimbrial protein might'serve as a model of effective vaccines against periodontitis.

Immunizing experimental animals with an outer membrane preparation and polysaccharide-protein conjugate isolated from P.gingivalis induces elevated levels of specific antibody and provides protection against the progression of periodontal disease (20). Immunization with hemagglutinin is also as efficient as that with whole cells in reducing recovery from P.gingivalis infection in ligated hamsters (13). O'Brien-Simpson et al. reported that the, incidence of secondary lesions was reduced by inununization with lithium diiodosalicylate extracts of membranes from P.gingivalis in the mouse abscess model but tissue invasion was not elicited. They also demonstrated that immunization with a purified $75-\mathrm{kDa}$ outer membrane protein reduces the activities of collagenase, gelatinase and cysteine proteases in gingival tissue. However, it did not prevent periodontal bone loss (21).

Okuda et al. showed that the antibodies elicited against fimbriae composed of a $54 \mathrm{kDa}$ protein derived from A. actinomycetemcomitans 310 -a protect against continued infection by this microorganism (22). They postulated that the $\operatorname{IgG}$ responses to fimbria antigen elicited by the initial contact with A. actinomycetemcomitans played an 
important role for eliminating organisms from the periodontal pockets of patients harboring high $\operatorname{IgG}$ antibody avidity.

\section{Antigen: Synthetic peptides}

Mapping the adhesion, T-cell and B-cell epitopes is essential for investigating synthetic peptide vaccines (23). T and Bcell epitopes are recognized by $\mathrm{T}$ cells and $\mathrm{B}$ cells, respectively. Adhesion epitope mediates adherence between bacteria and host tissue through a ligand-receptor interaction. It is important to design a synthetic peptide vaccine in which antigenicity does not imply immunogenicity. Since $\operatorname{IgG}$ and secretary $\operatorname{IgA}$ may play a role in preventing bacterial adhesion to salivary glycoproteins or mucosal receptors, adhesion epitopes are also indispensable to the immune response elicited by synthetic peptide vaccines. Small antigenic peptides are normally poorly immunogenic, and it is therefore necessary for small peptides to be added a carrier molecule for inducing an immune response.

Recombinant P. gingivalis HSP60 was produced and purified from P. gingivalis GroEL gene (24). Rats were immunized with P. gingivalis HSP60, and experimental alveolar bone loss was induced by infection with multiple periodontopathogenic bacteria. There was a very strong inverse relationship between postimmune anti-P. gingivalis HSP immunoglobulin $G(\operatorname{Ig} G)$ levels and the amount of alveolar bone loss induced by either $\mathrm{P}$. gingivalis or multiple bacterial infection $(\mathrm{p}=0.007)$. Polymerase chain reaction data indicated that the vaccine successfully eradicated the multiple pathogenic species.

Synthetic peptides based on the protein structure of fimbrillin inhibit the adhesion of $\mathrm{P}$. gingivalis to saliva-coated hydroxyapatite crystals in vitro and their binding domains are located at the carboxyl-terminal region (25). Furthermore, gingival tissue enzyme levels and horizontal bone loss were reduced by immunization with a 20 mer synthetic fimbrial peptide in the gnotobiotic rat model. Bran et al. investigated the linear immunogenic and antigenic structure of P.gingivalis fimbrillin and identified the antigenic determinant in native fimbrillin as residues 99-110. Furthermore, they suggested that peptide immunogens would be effective as vaccines since they could adopt a more native conformation to produce effective antibodies.

\section{Passive immunization}

Chronic disease is not generally an indication for passive immunization by the repeated administration of a xenogeneic immunoglobulin. However, passive immunization against periodontal diseases has been attempted because of the success of active and passive immunization against P.gingivalis and S. mutans, respectively. Passive immunization of humans using $\mathrm{P}$. gingivalis monoclonal antibodies temporarily prevents colonization of $\mathrm{P}$. gingivalis (5).

Okuda et al. reported that repeated passive immunization with rabbit antiserum to P.gingivalis hemagglutinin into the oral cavities of the hamsters reduced colonization by exogenous P.gingivalis in the periodontal region (13). Furthermore, passive immunization with monoclonal antibodies against P.gingivalis effect selectively prevents recolonization by this organism in humans. However, the periodontal health of patients was not significantly improved in either immunized or sham-immunized patients at 6 and 12 months.

A. actinomycetemcomitans-specific T-cell clone isolated and adoptively transferred into rats, elevated serum $\operatorname{IgG}$ and $\operatorname{IgM}$ antibodies to $\mathrm{A}$. actinomycetemcomitans and significantly decreased bone loss. Thus, T-cell regulation seems to affect periodontal disease and $\mathrm{T}$ helper cells apparently interfered with periodontal bone loss.

Hisashi Takiguchi et al, developed a panel of monoclonal antibodies by immunizing mice with purified $\mathrm{r} 40-\mathrm{kDa}$ OMP (26). The objective of their study was to determine the bactericidal activity on P.gingivalis by the IgG1 monoclonal antibody Pg-OMP A2. The results showed that in the presence of complement, Pg-OMP A2 was lethal to P.gingivalis 381 as well as to the more virulent P.gingivalis strains. Using component deficient compliment, they determined that Pg-OMP A2 killed P.gingivalis by activating both the classical and alternative complement pathways.

They concluded that Pg-OMP A2 has an in vitro complement-mediated bactericidal activity to P.gingivalis. Pg-OMP A2 may contribute to the development of a local immunotherapy that can be applied in the gingival crevice of a patient with P.gingivalis related periodontitis, or be a vaccine candidate.

\section{LIMITATIONS OF THE PERIODONTAL VACCINE}

First of all the complexity of the periodontopathic bacteria might be a problem as a substantial number of bacteria appear to be involved in periodontal disease. So determination of an antigen for the vaccine may pose as a major limitation.

Some more of the serious complications may stem from the vaccine or from the patient. Vaccines may be contaminated with unwanted proteins or toxins, or even live viruses. Supposedly killed vaccines may not have been properly killed; attenuated vaccines may revert to the wild type (1). The patient may be hypersensitive to minute amounts of contaminating proteins, or immuno-compromised, in which case any living vaccine is usually contraindicated. 
Having been somewhat ignored in the early days, safety has now become an overriding consideration. It is of course a relative term, minor local pains or swelling at the injection site, and even mild fever, being generally acceptable, although the public (led by legal profession) is becoming increasingly aware of the possibilities of profitable litigation.

\section{FUTURE DIRECTIONS}

Subunit vaccines have been developed based on viral and bacterial peptides or plasmid vectors. In fact, DNA vaccines that were first described less than five years ago have already progressed to phase I clinical trials in healthy adult humans. They might induce immunity to numerous agents, including periodontopathic bacteria, following confirmation of their safety. DNA vaccines offer several distinct advantages. Firstly, DNA vaccines can be manufactured more easily than vaccines consisting of an attenuated pathogen, an outer or internal protein or a recombinant protein. The second advantage is that since DNA is stable by nature and resistant to extremes of temperature, storage, transport and distribution, it might be highly practical. The third advantage of vaccination with DNA is the simplicity of changing the sequences encoding antigenic proteins by means of mutagenesis and of adding heterologous epitopes by basic molecular genetics. The immunogenicity of the modified protein may be directly assessed following an injection of DNA vaccine.

DNA plasmids encoding a gene required for antigen production are transferred by intramuscular needle injection without adjuvant. Alternatively, intradermal particle bombardment is also effective. Polynucleotides encoding pathogenic protein should not be injected. If DNA uptake is efficient, a single intramuscular injection can elicit a strong and sustained immune response. Antibodies are induced by immune responses. The immunoglobulin is ultimately an IgG, indicating a T-cell dependent class switch. The responses include not only antibody induction and T-cell activation with cytokine secretion, but also the production of cytoxic $\mathrm{T}$ cells.

DNA vaccination has been studied in animals. Most of the investigated pathogens have been viruses, for instance bovine herpes virus, hepatitis B virus, hepatitis C virus, herpes simplex virus, human immunodeficiency virus-1, influenza virus and lymphocytic choriomeningitis virus. Furthermore, some pathogenic bacteria have also been investigated. Lowrie et al. demonstrated that expression of the gene for a single mycobacterial antigen (Mycobacterium leprae hsp 65) in adult BALB/c mice caused substantial cell-mediated protection against challenge with Mycobacterium tuberculosis. Some genes from periodontopathic bacteria have been cloned and these genes could be used as a vaccine to protect against periodontitis (27-30). DNA vaccines have distinct potential for preventing various infectious diseases, including periodontal disease, in humans.

\section{REFERENCES}

1. Roitt, Brostoff, Male. Text book of Immunology, Fourth Edition, 1998.

2. Grossi SG, Genoc RJ, Machtei EE, Ho AW, Koch G, Dunford R, et al. Assessment of risk for periodontal disease. II. Risk indicators for alveolar bone loss. J Periodontol 1995;66:23-29.

3. Grossi SG, Zambon 11, Ho AW, Koch G, Dunford RG, Machtei EE, et al. Assessment of risk for periodontal disease. I. Risk indicators for attachment loss. J Periodontol 1994;65:260-267.

4. Page RC, Genco RJ. Mucosal immunity and periodontitis: Mucosal vaccines. San Diego: Academic Press 1996;437-449.

5. Persson GR.Immune responses and vaccination against periodontal infections. J Clin Periodontol 2005;32(Suppl 6):54-56.

6. McArthur WP, Magnusson I, Marks RG, Clark WB. Modulation of colonization by black-pigmented Bacterial species in squirrel monkeys by immunization with Bacteroides gingivalis. Infect Immun 1989;57:2313-2317.

7. Persson GR, Engel LD, Whitney CW, Weinberg A, Monda B1, Darveau RP, et al. Macaca Fascicularis as a model in which to assess the safety and efficacy of a vaccine for periodontitis. Oral Microbiol Immunol 1994;9:104-111.

8. Page RC, Schroeder HE. Periodontitis in man and other animals. Basel: Karger 1982.

9. Klausen B. Microbiological and immunological aspects of experimental periodontal disease in rats: a review article. $J$ Periodontol 1991;62:59-73.

10. Kesavalu L, Ebersole IL, Machen RL, Holt SC. Porphyromonas gingiva/is virulence in mice: induction of immunity to bacterial components. Infect Immun 1992;60:1455-1464.

11. Klausen B, Evans RT, Ramamurhty NS, Golub LM, Sfintescu C, Lee IY, et al. Periodontal bone level and gingival proteinase activity in gnotobiotic rats immunized with Bacteroides gingiva/is. Oral Microbiol Immunol 1991;6:193-201.

12. Genco CA, Kapczynski DR, Cutler CW, Arko RJ, Arnold RF. Influence of immunization 01\}-Porphyromonas gingiva/is, colonization and invasion in the mouse chamber model. Infect Immun 1992;60:1447-1454.

13. Okuda K, Kato T, Naito Y, Takazoe I, Kikuchi Y, Nakamura T, et al. Protective efficacy of active and passive immunizations against experimental infection with Bacteroides gingiva/is in ligated hamsters. J Dent Res 1998;67:807-811.

14. Persson GR, Engel LD, Whitney C, Darveau RP, Weinberg A, Brunsvold $\mathrm{M}$, et al. Immunization against Porphyromonas gingiva/ is inhibits progression of experimental periodontitis in nonhuman primates. Infect Immun 1994;62:1026-1031.

15. Hardham J, Sfintescu C, Evans RT. Evaluation of cross-protection by immunization with an experimental trivalent companion animal periodontitis vaccine in the mouse periodontitis model. J Vet Dent 2008;25(1): 23-27.

16. Page RC. Vaccination and periodontitis: myth or reality. J Int Acad Periodontol 2000;2(2): 31-43.

17. Ebersole JL, Brunsvold M, Steffensen B, Wood R Holt SC. Effects of immunization with PorRhyromonas gingiva/is and Prevote//a intermedia on progression of ligature-induced periodontitis in the nonhuman primate Macaca fascicu/aris. Infect Immun 1991;59: 3351-3359.

18. Okuda J, Slots J, Genco RJ. Bacteroides gingivalis. Bacteroides asaccharolyticus, and Bacteroides melaninogenicus subspecies: cell surface morphology and adherence to erythrocytes and human buccal epithelial cells. CUff Microbiol 1981;6:7-12.

19. Evans RT, Klausen B, Sojar HT, Bedi GS, Sfintescu C, Ramamurthy NS, et al. Inununization with Porphyromonas (Bactero.ides) gingivalis fimbriae against periodontal destruction. Infect Immun 1992;2926-2935. 
PERIODONTAL VACCINE: A NEW PARADIGM FOR PREVENTION OF PERIODONTAL DISEASES

20. Schifferle RE, Reddy MS, Zambon JJ, Genco RJ. Characterization of a polysaccharide antigen from Bacteroides gingivalis. J Immunol 1989;143:3035-3042.

21. O'Brien-Simpson NM, Pathirana RD, Paolini RA, Chen YY, Veith $\mathrm{PD}, \mathrm{Tam} \mathrm{V}$, et al. An immune response directed to proteinase and adhesion functional epitopes protects against P.gingivalis- induced periodontal bone loss. J Immunol 2005;175(6):3980-3989.

22. Okuda K, Saito A, Hirai K, Harano K, Kato T. The role of antibodies to periodontal organisms. In: Genco RJ Parkh-ill R, Ed. Molecular basis for pathogenesis and molecular targeting in periodontal diseases. Buffalo: SUNY at Buffalo, Department of Oral Biology 1993:40.

23. Lehner T, Ma JKC, Walker P, Childerstone A, Todryk S, Kendal H, et al. Tcell and B-cell epitope mapping and construction of peptide vaccines. In: Genco RJ, Hamada S, Lehner T, McGhee JR, Mergenhagen S, ed. Molecular pathogenesis of periodontal disease. Washington, DC: ASM Press, 1994;279-292.

24. Lee JY, Yi NN, Kim US, Choi JS, Kim SJ, Choi Jl. Porphyromonas gingivalis heat shock protein vaccine reduces the alveolar bone loss induced by multiple periodontopathogenic bacteria. $J$ Periodontal Res 2006;41(1):10-14.
25. Lee JY, Sojar HT, Bedi GS, Genco RJ. Synthetic peptides analogous to the fimbrillin sequence inhibit adherence of Porphyromonas gingivalis. Infect Immun 1992;60:1662-1670.

26. Hisashi Takiguchi, Mitsunobu Katoh, Shigeno Saito and Yoshimitsu Abiko. Bactericidal Activity of a Monoclonal Antibody Against Recombinant $40-k D a$ Outer Membrane Protein of P.gingivalis. $J$ Periodontal 2000;71:368-375.

27. Hayakawa M, Abiko Y, Ito T, Sasahara H, Yamano H, Takiguchi H. Gene cloning of Porphyromonas gingivalis specific antigens recognized by serum of adult periodontitis patient. Int $J$ Biochem 1992;24:945-950.

28. Joe A, Yamamoto A, McBride BC. Characterization of recombinant and native forms of a cell surface antigen of Porphyromonas (Bacteroides) gingivalis. Infect Immun 1993;61:3294-3303.

29. Miyamoto M, Noji S, Kokeguchi S, Kato K, Kurihara H, Murayama $\mathrm{Y}$, et al. Molecular cloning and sequence analysis of antigen gene tdpA of Treponema denticola. Infect Immun 1991;59:1941-1947.

30. Ogawa T, Mori H, Yasuda K, Hasegawa M. Molecular cloning and characterization of the genes encoding the immunore active major cell-surface proteins of Porphyromonas gingivalis. FEMS Microbiol Lett 1994;120:23-30. 